\title{
BMJ Open Implementing an evidence-based somatic acupressure intervention in breast cancer survivors with the symptom cluster of fatigue, sleep disturbance and depression: study protocol of a phase II randomised controlled trial
}

\author{
Tao Wang (D) , ${ }^{1}$ Jing-Yu (Benjamin) Tan (1) , ${ }^{2}$ Xian-Liang Liu, ${ }^{1}$ Si-Lin Zheng, ${ }^{3}$ \\ Isabella Zhao, ${ }^{1,4}$ Sabina Eliseeva, ${ }^{1,5}$ Mary Janice Polotan, ${ }^{1,5}$ Hui-Lin Cheng (D) ,1,6 \\ Hou-Qiang Huang ${ }^{3}$
}

To cite: Wang T, Tan J-YB, Liu X-L, et al. Implementing an evidence-based somatic acupressure intervention in breast cancer survivors with the symptom cluster of fatigue, sleep disturbance and depression: study protocol of a phase II randomised controlled trial. BMJ Open 2022;12:e054597. doi:10.1136/ bmjopen-2021-054597

- Prepublication history and additional supplemental material for this paper are available online. To view these files, please visit the journal online (http://dx.doi.org/10.1136/ bmjopen-2021-054597)

Received 21 June 2021 Accepted 20 December 2021

Check for updates

(c) Author(s) (or their employer(s)) 2022. Re-use permitted under CC BY-NC. No commercial re-use. See rights and permissions. Published by BMJ.

For numbered affiliations see end of article.

Correspondence to Professor Jing-Yu (Benjamin) Tan; benjamin.tan@cdu.edu.au

\section{ABSTRACT}

Introduction The fatigue-sleep disturbance-depression (FSD) symptom cluster, as one of the most common symptom clusters in breast cancer (BC) survivors, can significantly decrease patients' quality of life. Since the management of the FSD symptom cluster has been unsatisfactory with the use of pharmacological treatments alone, non-pharmacological approaches have, therefore, been recommended. Somatic acupressure (SA) is a promising approach given its potential benefits of cancer-related symptom alleviation and the convenience of self-practice. However, research evidence on using acupressure to manage the FSD symptom cluster has been limited. The proposed trial aims to examine the feasibility and preliminary effects of an evidence-based SA intervention for FSD symptom cluster management in BC survivors.

Methods and analysis This study will be a phase II randomised controlled trial with three study arms and 1:1:1 allocation. Fifty-one early-stage BC survivors who are experiencing the FSD symptom cluster will be randomly assigned to a true SA group, a sham SA group or a usual care group. All participants will receive an education booklet regarding FSD symptom cluster management advice as the usual care package. The participants in the true SA group will additionally receive a 7-week self-administered SA intervention. The participants in the sham SA group will additionally receive self-administered light acupressure at non-acupoints with the same frequency, session and duration as the true SA group. The primary outcomes will be feasibility outcomes related to subject recruitment and completion of study questionnaires and interventions. The secondary outcomes will be the effects of SA on fatigue, sleep disturbance, depression and quality of life. Descriptive statistics will be used to present all the outcomes. The secondary outcomes will be analysed using an intent-totreat approach.

\section{Strengths and limitations of this study}

- The design of this study will follow the Medical Research Council Framework for Developing and Evaluating Complex Interventions, which will improve the study's fit with clinical practice in a local context and increase its likelihood of success.

- This study will use an evidence-based and researchinformed method to develop the somatic acupressure (SA) intervention protocol on the basis of widely recognised theories, best available research evidence and practice standards, and the consensus of an expert panel.

- This trial will adopt a sham SA comparison, which will enable the measurement of potential placebo effects of the SA interventions.

- This study will be challenging to maintain a successful blind design among the participants due to the visible nature of the intervention.

Ethics and dissemination Ethical approvals of this trial have been granted by the Human Research Ethics Committee at Charles Darwin University (H19017) and the Clinical Trial Ethics Committee at The Affiliated Hospital of Southwest Medical University (KY2019039). Findings from this trial will be published in peer-reviewed journals and presented at professional conferences.

Trial registration number This trial was registered at ClinicalTrials.gov and the registration number is NCT04118140, with the stage at Recruiting.

\section{INTRODUCTION}

As the most common cancer diagnosis among females worldwide, breast cancer (BC) has become a curable condition in $70 \%-80 \%$ 
of early-stage survivors, ${ }^{1}$ with a 5 -year survival rate up to $92.2 \% .^{2}$ However, substantial late effects and physical symptoms that are associated with cancer and cancerrelated treatment, such as fatigue, pain, sleep disturbance and lymphoedema, are still a prominent concern for BC survivors. ${ }^{3}$ Fatigue is the most common symptom, which occurs in more than $75 \%$ of BC survivors, ${ }^{45}$ persisting for years after completing treatment. ${ }^{6}$ Sleep disturbance and depression are another two frequently identified symptoms among BC survivors, with the incidence of $47 \%-65 \%$ and $25 \%-39 \%$, respectively, in those who have completed primary and adjuvant cancer treatment. ${ }^{7-10}$ Cancer-related fatigue, sleep disturbance and depression do not present as isolated conditions but often co-occur as a symptom cluster. ${ }^{11}$ Fatigue has been identified as the core symptom contributing to the symptom cluster, which can predict the symptoms of sleep disturbance and depression. ${ }^{11} 12$ The onset of sleep disturbance also contributes to more severe fatigue, and vice versa, while more severe depressive conditions have been identified as closely linked with more deteriorated sleep problems. ${ }^{13}$ The fatigue-sleep disturbance-depression (FSD) symptom cluster is therefore one of the most frequently reported symptom clusters in BC survivors, which can deteriorate survivors' physical and psychological well-being, lead to increased medical complications, financial burden, and healthcare resources utilisation, and even impede survivors' compliance with treatment and follow-up and shorten their survival. ${ }^{6} 11$ 14-17

Tailored pharmaceutical agents for FSD symptom cluster management in BC have been lacking, ${ }^{15}$ and the available possibly effective pharmaceutical agents mainly focus on alleviating a single symptom rather than the entire symptom cluster. For example, the effects of melatonin on cancer-related sleep disturbance, fatigue, and depression have been controversial, with research evidence identified only in several small-scale trials. ${ }^{151819}$ Meanwhile, precautions should be emphasised when applying pharmaceutical agents to cancer patients considering the possibility of drug-related adverse events and the potential drug interactions with concurrent antineoplastic regimens. ${ }^{15}$ To address this issue, various non-pharmacological approaches, such as cognitive-behavioural therapy (CBT), physical exercise, yoga and other mindfulnessbased arts interventions (eg, meditation), have been applied in practice as a combination approach with pharmaceutical agents for the management of the individual symptoms of fatigue, sleep disturbance and depression in cancer survivors, with beneficial effects demonstrated in clinical research. ${ }^{1520-22}$ Approaches such as CBT, yoga and other mindfulness-based arts interventions are time, cost and energy-consuming, with an extensive requirement of professional support. Professional-supervised approaches also limit the space for the self-management of symptoms at home in the long run. Physical exercise itself may contribute to recruitment bias as fatigue is the core symptom of the FSD symptom cluster, which may hinder study participation. ${ }^{6}$ Other non-pharmacological approaches that can be self-practised by cancer survivors to facilitate better self-management of the FSD symptom cluster in the long term is warranted.

Somatic acupoint stimulation, including acupuncture and acupressure, has been commonly practised to manage a wide range of health conditions. The Yin-Yang theory and Zang-Fu organs and meridians theory have highlighted the underpinning mechanisms of acupoint stimulation of specific acupoints, which can facilitate the circulation of qi and blood through typical body regions and Zang-Fu organs, dredging the meridians, balancing Yin and Yang, regulating Zang-Fu functions ${ }^{23} 24$ and eliminating pathogenic conditions. The potential biological mechanisms of acupoint stimulation, which involve the regulation of proinflammatory cytokines, highlight the promising role of acupoint stimulation for FSD symptom cluster management as the onset of fatigue within the cluster is believed to be closely linked with proinflammatory cytokines. ${ }^{13}$ Existing clinical studies have supported the promising role of acupoint stimulation for the single symptom management of fatigue, sleep disturbance and depression in cancer patients, ${ }^{20} 2123$ although relevant evidence has been limited given the unsatisfactory methodological quality and small sample size with underpowered data analysis in some studies. Studies that have used acupoint stimulation to manage symptom clusters in cancer patients are scant. According to a systematic review on non-pharmacological interventions for symptom clusters management in cancer patients published in $2020,{ }^{25}$ only 3 of the 13 included randomised controlled trials (RCTs) used acupoint stimulation to manage symptom clusters. Of the three RCTs, none focused on the FSD symptom cluster and none adopted a sham comparison to examine the placebo effects of acupoint stimulation. ${ }^{25}$ In $2020^{26}$ and $2021,{ }^{27}$ two other pilot RCTs were published, and the findings indicated that self-acupressure was a feasible and acceptable approach for symptom cluster management in cancer patients. However, only one study adopted a three-arm randomised sham-controlled design, ${ }^{27}$ and neither of the two studies specifically focused on BC survivors experiencing the FSD symptom cluster, with one study focusing on the symptoms of fatigue, insomnia and/or pain in advanced cancer patients ${ }^{26}$ and the other one on the insomnia-depression-anxiety symptom cluster in cancer patients undergoing chemotherapy. ${ }^{27}$ Taken together, there is a need and possibility to develop an acupoint stimulation intervention that can be adopted by BC survivors to comprehensively manage the FSD symptom cluster.

Compared with acupuncture, which is administered by qualified practitioners and is invasive in nature, with potentially serious adverse events, non-invasive somatic acupressure (SA) is easy to learn and is a relatively safe intervention for the self-management of cancer symptoms in the long run. This study, therefore, proposes to evaluate the feasibility and potential effects of an evidence-based SA intervention for managing the FSD symptom cluster in BC survivors through a phase II RCT. 
The Medical Research Council (MRC) Framework for Developing and Evaluating Complex Interventions (the MRC Framework) ${ }^{28}$ will be used to guide the design and methodological procedures of this study. The protocol of this trial has been reported in accordance with the Standard Protocol Items: Recommendations for Interventional Trials Checklist. ${ }^{29}$

\section{AIMS AND OBJECTIVES}

This study aims to examine the feasibility of an evidencebased SA intervention and its potential effects on the FSD symptom cluster and quality of life (QoL) in female BC survivors through a phase II RCT. The specific objectives of this RCT are:

1. To pilot the methodological procedures of the RCT using the SA intervention to manage the FSD symptom cluster in BC survivors.

2. To estimate the eligibility rate, recruitment rate, retention rate and attrition rate of the RCT subject recruitment process.

3. To evaluate the feasibility and acceptability of the SA intervention and the study questionnaires.

4. To examine the potential adverse events of SA.
5. To examine the potential effects of SA on the FSD symptom cluster and QoL in BC survivors.

\section{STUDY DESIGN}

This study will be a phase II three-arm sham-controlled RCT. The study's design and process are presented in figure 1. The timeline for subject enrolment, intervention delivery, and data collection and assessments is shown in table 1 .

\section{METHODS: PARTICIPANTS, INTERVENTIONS AND OUTCOMES Study setting}

This RCT will be conducted in a large tertiary medical centre, the Affiliated Hospital of Southwest Medical University, in Luzhou, Sichuan, China.

\section{Participants and sample size}

Female BC survivors who meet the following inclusion criteria will be recruited: (1) confirmed BC diagnosis from stage I to IIIa (early-stage BC survivors without any distant metastasis); (2) have experienced at least a moderate level of the FSD symptom cluster, with a score

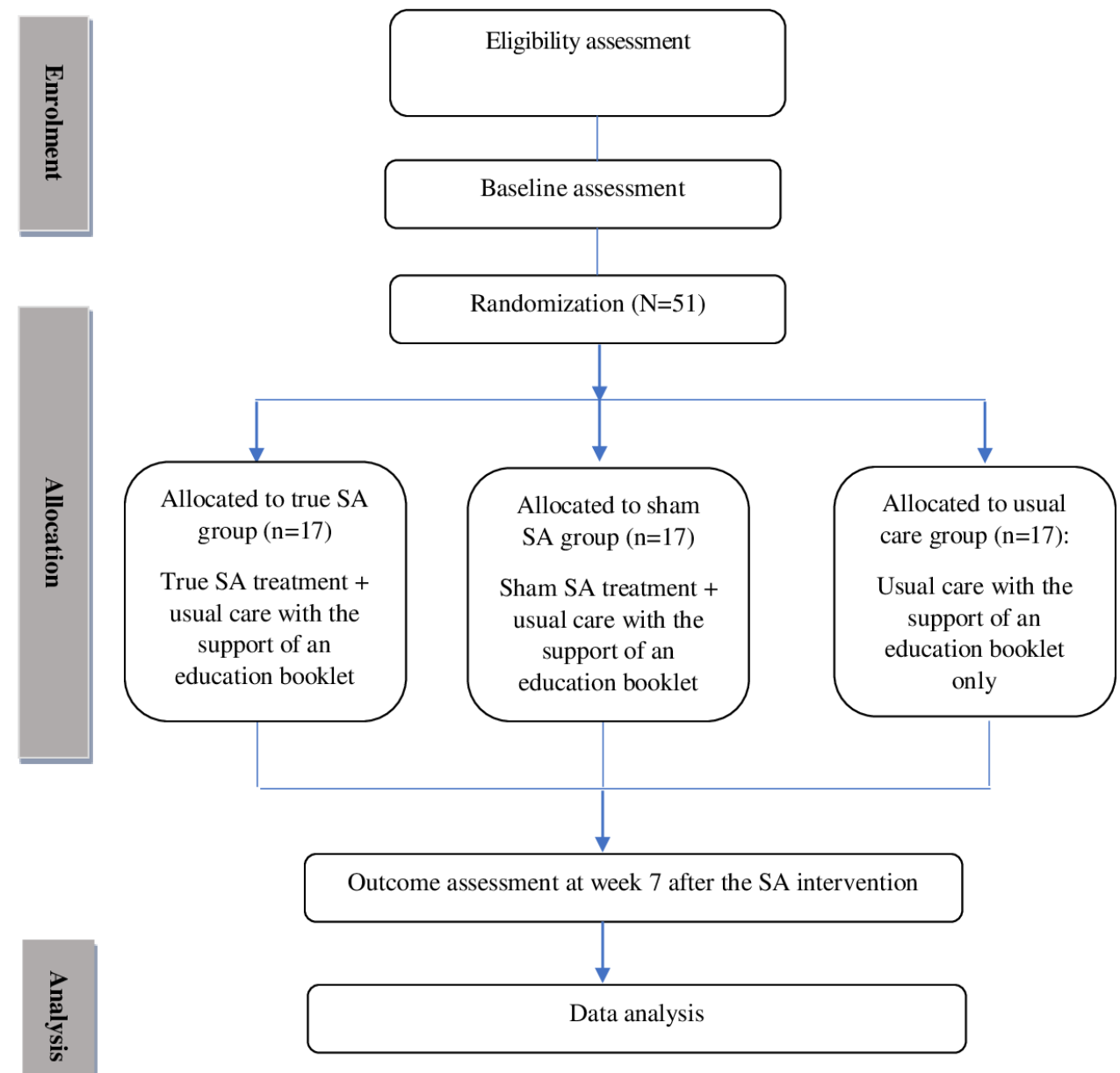

Figure 1 The CONSORT flow chart of this trial. CONSORT, Consolidated Standards of Reporting Trials; SA, somatic acupressure. 
Table 1 Time schedule for enrolment, interventions and outcome assessment

\section{Study period}

\begin{tabular}{|c|c|c|c|c|}
\hline & \multirow[b]{2}{*}{$\begin{array}{l}\text { Enrolment/recruitment } \\
\\
\text { Week }-1\end{array}$} & \multirow[b]{2}{*}{$\begin{array}{l}\text { Allocation } \\
\text { Week } 0\end{array}$} & \multicolumn{2}{|l|}{ After allocation } \\
\hline & & & $\begin{array}{l}\text { Weeks 1-7 (during } \\
\text { intervention) }\end{array}$ & $\begin{array}{l}\text { Week } 7 \\
\text { (immediately } \\
\text { after } \\
\text { completion } \\
\text { of the } \\
\text { intervention) }\end{array}$ \\
\hline Informed consent & $x$ & & & \\
\hline Participants' characteristics & $x$ & & & \\
\hline Allocation & & $x$ & & \\
\hline \multicolumn{5}{|l|}{ Interventions } \\
\hline $\begin{array}{l}\text { Feasibility of participant } \\
\text { recruitment }\end{array}$ & & & & $x$ \\
\hline $\begin{array}{l}\text { Feasibility of the } \\
\text { questionnaires }\end{array}$ & & & & $x$ \\
\hline $\begin{array}{l}\text { Feasibility and acceptability of } \\
\text { the interventions }\end{array}$ & & & & $x$ \\
\hline $\begin{array}{l}\text { Adverse events of the } \\
\text { intervention }\end{array}$ & & & $x$ & \\
\hline MFI & $x$ & & & $x$ \\
\hline HADS-D & $x$ & & & $x$ \\
\hline
\end{tabular}

FACT-B, Functional Assessment of Cancer Therapy-Breast; HADS, Hospital Anxiety and Depression Scale; MFI, Multidimensional Fatigue Inventory; PSQI, Pittsburgh Sleep Quality Index.

of $\geq 4$ on a $0-10$ Numeric Rating Scale ( $0=$ 'no symptom', $10=$ 'worst symptom') for all three symptoms (fatigue, sleep disturbance and depression) during the previous 1 month; (3) have completed adjuvant chemotherapy for at least 1 month and up to 3 years (to capture persistent symptoms); (4) have no scheduled chemotherapy and radiotherapy during the study period and (5) willing to participate in this RCT with written informed consent.

Potential participants will be excluded if they meet any of the following exclusion criteria: (1) presently taking pharmaceutical agents to treat fatigue, sleep disturbance or depression, such as antidepressant medications, psychostimulants or hypnotics; (2) extremely weak and/ or have cognitive impairment that make them unable (or would make it difficult for them) to follow the study procedures and instructions; (3) have received any type of SA treatment during the previous 6 months and (4) presently participating in any other research projects.

Julious $^{30}$ recommended that 12 subjects per group is necessary to justify a study's feasibility and the precision of the mean and variance. Given that the primary objective of the proposed study is to evaluate the feasibility and acceptability of the RCTs methodological procedure, intervention protocol and questionnaires, a sample size of 12 per group will therefore be considered. According to one previous study, ${ }^{31}$ the completion rate of 6 weeks of daily self-administered acupressure to manage cancer-related fatigue in BC survivors was $73.4 \%$. Given that the current study will consist of 7 weeks of daily selfadministered acupressure to measure the FSD symptom cluster in BC survivors, a possible attrition rate of $30 \%$ will be considered.The final sample size will be 51 , with 17 in each group.

\section{Recruitment and consent}

The potential participants will be recruited via the outpatient clinic at the study hospital as identified by oncology nurses, and those who are interested in this study will be referred to the research assistant. Then, the research assistant will approach those patients in person to further screen their eligibility based on the inclusion and exclusion criteria and invite the eligible participants 
to participate in the study. If the participants show an interest, an information sheet will be provided to them detailing the study's aim and procedures. All potential participants will be informed that they can refuse to participate or withdraw at any time without any consequences. If the participants agree to participate in the study, a written informed consent will be required.

\section{Randomisation}

To maintain an equal sample size across study arms, a block randomisation method will be used in the proposed study, with a 1:1:1 ratio, via the online application Research Randomiser (https://www.randomizer. $\mathrm{org} /$ ). The randomisation sequence will be generated by an independent statistician who will not be involved in any part of this study, including data analysis.

\section{Concealment}

Allocation concealment will be conducted to minimise the risk of selection bias. The allocation sequence will be kept by the independent statistician who will conduct the sequence generation. Group assignment will also be performed independently by the statistician. Once an eligible participant consents to participate in the study and completes the baseline assessment, the research assistant will call the statistician to determine the participant's group assignment.

\section{Blinding}

Considering that all the participants will be those who have no experience of SA treatment, it is therefore feasible to perform a partial blind design for the participants in the true and sham intervention groups. The blinding of outcome assessments will also be achieved in the true and sham intervention groups as all the questionnaires will be patient reported and the participants themselves will be the outcome assessors. Data analysis will be conducted by an independent statistician who will be blinded to group allocation.

\section{Study arms and interventions}

The proposed study will include three arms: the true SA group, the sham SA group and the usual care group. All participants will receive usual care from their treating clinicians with the support of an education booklet detailing knowledge of $\mathrm{BC}$ and general advice for managing fatigue, sleep disturbance and depression as the usual care package. The participants in the true and sham SA groups will receive an additional true and sham SA intervention, respectively.

\section{Explanation for choosing comparators}

Acupoint stimulation therapy has been universally believed to be associated with some non-specific treatment effects (placebo effects). ${ }^{32}$ To examine the specific treatment effects of SA, a sham SA group will therefore be introduced as an appropriate comparison to distinguish the specific effects of SA from its non-specific (placebo) effects. Meanwhile, a usual care comparison without any intervention will be designed as the third arm to further assess the size of the placebo effects in the sham SA intervention.

\section{Intervention description}

\section{True SA group}

The participants allocated to the true SA group will receive a 7-week self-administered SA intervention, which will be determined based on existing research evidence extracted from systematic reviews (three of which were completed by our study team, with two published ${ }^{33} 34$ and the other one under review), relevant theories (the inflammatory theory, the Yin-Yang theory and the Zang-Fu organs and meridians theory), ${ }^{2324}$ practice standards ${ }^{35} 36$ and experts' consensus. Eleven acupoints will be used as the therapeutic acupoints formula: Zusanli (ST36), Sanyinjiao (SP6), Taixi (KI3), Hegu (LI4), Neiguan (PC6), Shenmen (HT7), Baihui (GV20), Qihai (CV6), Guanyuan (CV4), Yintang (EX-HN3) and Taichong (LR3). The treatment will be scheduled once per day, with each session lasting about $36 \mathrm{~min}$, pressing each acupoint for $2 \mathrm{~min}$ using the participants' finger (seven of the eleven acupoints are bilateral). Daily acupressure can be conducted at any time based on the participants' preference and convenience. The intensity of the stimulation will be determined by the achievement of the 'Deqi' sensation. The practice standards of finger acupressure ${ }^{37}$ will be used to guide the implementation of acupressure in this study, which will involve the skills of pointing, pressing and kneading. To ensure that the participants fully master the SA skills, each patient will receive SA training prior to the commencement of the intervention until they satisfy the research assistant's assessment. In addition, a home learning package in the format of visual materials will be provided to the participants to support their self-practice of SA at home. The procedures of the development and validation of the SA intervention protocol will be reported in a separate methodological paper, which will be submitted to a journal for publication. As this is a home-based self-acupressure intervention, weekly telephone follow-ups will be conducted by the research assistant during the entire study period to remind the participants to complete the SA daily and to address the participants' questions in relation to practising SA.

\section{Sham SA group}

The participants allocated to the sham SA group will receive a self-administered sham SA, the protocol of which will be developed based on evidence retrieved from two systematic reviews on sham acupoint stimulation designs ${ }^{38} 39$ and experts' consensus. To achieve a satisfactory blind design of the participants and to minimise the potential therapeutic effects of the sham SA, non-acupoints located $1-3 \mathrm{~cm}$ away from the acupoints used in the true SA group but away from the meridians ${ }^{38}$ will be used in the sham SA group. Frequency, session, and total duration of the sham SA will be the same as 
those for the true SA group. Light acupressure on nonacupoints without the sensation of 'Deqi' will be employed to further minimise the therapeutic effects of the sham SA. Weekly telephone follow-ups will also be conducted by the research assistant during the entire study period. On completion of the study, the participants in this group will have the opportunity to receive the true SA intervention on a voluntary basis.

\section{Usual care group}

The participants allocated to the usual care group will only receive usual care from their treating doctors, with the support of an education booklet for cancer symptom management. All information and recommendations listed in this booklet will be retrieved from relevant national guidelines (eg, the National Comprehensive Cancer Network Clinical Practice Guidelines in Oncology ${ }^{40}$ ), local health authorities (eg, Department of Health at the Government of Western Australia ${ }^{41}$ ) and peer-reviewed research articles. ${ }^{5} 4243$ On completion of the study, the participants in this group will have the opportunity to receive the true SA intervention on a voluntary basis.

\section{Intervention adherence and monitoring}

A specially designed daily $\log$ will be provided to the participants in the true and sham SA groups to record their daily practising of SA, including the date, time and duration of each SA session, and any difficulties and adverse events that occurred during the SA process. Moreover, to further enhance their adherence, weekly telephone call will be conducted by the research assistant during the entire study process to remind the participants in both the true and sham SA groups to complete the intervention daily and to address their questions in relation to practising SA. If participants withdraw, the reasons for withdrawal will be collected when possible, and relevant strategies will be proposed via research group discussion to minimise any further dropouts. To monitor the study's progress and adverse events related to the SA intervention, as well as to review the safety and quality of the data collected, principal investigator-led biweekly meetings with all other investigators, including the research assistant, will be conducted. If problems that can affect the study's implementation are identified, decisions on modifying the study's protocol will be made by the principal investigator via research team meetings; for any modifications, ethical approvals will be updated at both the principal investigator's institution and the study hospital before the implementation of the modified study protocol.

\section{Intervention fidelity}

To maintain intervention fidelity, the intervention protocol will be developed based on the best available research evidence identified in well-conducted systematic reviews and clinical trials, as well as relevant traditional Chinese medicine (TCM) theories and practice standards regarding the selection of acupoints, modality, intensity and technique and dosage of the intervention. The content validity of the intervention protocol will be examined via a panel of experts specialised in oncology and/or TCM. To ensure that the intervention protocol can be implemented as designed, intensive training of the research assistant will be performed prior to the commencement of the trial regarding acupressure skills, as well as verbal communication between the research assistant and the participants. A qualified acupuncture practitioner will be responsible for the training. After the training, an evaluation will be performed by the acupuncture practitioner to ensure that the research assistant has fully mastered the accurate location of the acupoints and acupressure skills. Professional support will be provided by the acupuncture practitioner throughout the study. During the period of study implementation, biweekly meetings will be held between the research assistant, acupuncture practitioner and principal investigator to discuss and solve any potential inconsistencies regarding the implementation of the intervention protocol as well as to monitor the intervention protocol delivery process to maintain internal validity. ${ }^{45}$ Regarding the reception of the intervention protocol (BC survivors in both the true and sham SA groups), each participant will receive acupressure training prior to the commencement of the intervention until they satisfy the research assistant's assessment. A return demonstration after the training will be performed to ensure that the study participants totally understand the intervention procedures and are able to follow the protocol to complete the intervention. Written and visual instructions on the intervention procedures, acupoint locations and acupressure skills will be given to the participants so that they can follow the instructions and perform acupressure at home.

\section{Patient and public involvement}

No patients or members of the public will be involved in the design, conduct, reporting or dissemination plans of our research.

\section{Outcome measures}

The primary outcomes will be all of the feasibility outcomes related to subject recruitment and the completion of the study questionnaires and interventions. The secondary outcomes will be clinical outcomes regarding the effects of SA on fatigue, sleep-disturbance, depression and QoL in BC survivors.

\section{Baseline assessment}

The participants' demographic and medical information will be collected via a self-designed baseline data assessment form and extracted from the participants' medical records. The demographic information will mainly include the participants' age, marital status, education background, occupation, religion, family income, place of residence and source of medical insurance. The medical information will include the participants' current cancer 
stage, date of confirming $\mathrm{BC}$ diagnosis, date and type of BC surgery and history of anticancer treatment such as scheduled chemotherapeutic agents and combinations.

Primary outcomes: feasibility outcomes

Feasibility of subject recruitment

The following feasibility outcomes regarding the subject recruitment process will be collected: (1) duration of completing subject recruitment; (2) eligibility rate (number of eligible participants/number of participants screened); (3) recruitment rate (number of participants who participated in the study/number of eligible participants); (4) retention rate (number of participants who completed the study/number of participants who enrolled in the study); (5) attrition rate (number of participants who dropped out after randomisation/number of participants who enrolled in the study) and (6) participants' feedback regarding why they discontinued the study. The following criteria will be set to determine the success of the proposed pilot study based on previous studies on self-acupressure for the management of cancer symptoms: eligibility rate $\geq 20 \%,{ }^{27}$ recruitment rate $\geq 70 \%,{ }^{46}$ retention rate $\geq 80 \%{ }^{2646}$ and attrition rate $\leq 30 \%$. ${ }^{26}$

\section{Feasibility of the study questionnaires}

Feasibility assessment of the study questionnaires will include: (1) item-level missing values in each questionnaire: the percentage of participants who did not answer a single item and (2) scale-level missing values in the questionnaire: the percentage of participants who did not answer at least one item in the whole questionnaire. The questionnaires that will be used in this study will be the Multidimensional Fatigue Inventory (MFI), the Pittsburgh Sleep Quality Index (PSQI), the Hospital Anxiety and Depression Scale (HADS) and the Functional Assessment of Cancer Therapy-Breast (FACT-B).

\section{Feasibility and acceptability of the study interventions}

The feasibility and acceptability of the study interventions will be assessed by: (1) the number of days that the participants performed the SA interventions, where the scheduled sessions will be 7 weeks of daily acupressure; (2) duration of each time of acupressure, where the scheduled time per session will be around $36 \mathrm{~min}$ and (3) participants' feedback and satisfaction with the SA interventions using a self-designed feedback form and follow-up semistructured interviews after the completion of the RCT. The participants' adherence to the intervention will be assessed by calculating the proportion of participants who followed the protocol and completed the required number of SA days and the duration of each session of acupressure $(\geq 70 \%) .{ }^{46}$ Semistructured interviews will be conducted by the research assistant, who has rich experience in conducting qualitative interviews, to explore the participants' experiences of participating in this study and receiving the intervention. A preliminary interview guide is presented in online supplemental appendix 1.

\section{Safety assessment}

Any adverse events that occurred during the intervention will be recorded by both the participants (via a daily $\log$ ) and the research assistant (via a case report form). If any adverse events occur, the research assistant and the acupuncture practitioner will approach and interview the relevant participant to collect more details about the adverse event. An adverse event report will then be completed by the research assistant and the acupuncture practitioner. The causality between the adverse event and the intervention will be assessed by the acupuncture practitioner based on his/her clinical experience.

\section{Secondary outcomes: clinical outcomes Fatigue}

The MFI (20 items) will be used to assess the participants' severity of fatigue. A higher score indicates more severe symptoms. ${ }^{47}$ The Chinese version of the MFI-20 has been demonstrated to be a reliable and valid instrument for Chinese cancer patients. ${ }^{47}$

\section{Sleep disturbance}

The PSQI will be used to assess the participants' sleep quality, which has seven domains, including subjective sleep quality, habitual sleep efficiency, sleep latency, sleep disturbances, sleep medication, sleep duration and daytime dysfunction. ${ }^{48}$ A higher score indicates poorer sleep quality. ${ }^{49}$ The PSQI has been tested as a reliable, valid, and sensitive instrument for measuring Chinese cancer patients' sleep quality. ${ }^{49}$

\section{Depression}

The HADS consists of two subscales, HADS-A for measuring anxiety and HADS-D for measuring depression. ${ }^{50}$ In this study, the depression subscale (HADS-D) will be used to measure the participants' depression. A higher score indicates a greater severity of depression. As a commonly used instrument for measuring cancer patients' depression, the HADS-D has been demonstrated to have well-documented psychometric properties. ${ }^{50}$

\section{Quality of life}

The participants' QoL will be measured by the FACT-B, which includes two major parts: the FACT-General scale (27 items) and the breast-cancer-specific subscale (10 items). The FACT-B has been demonstrated to have good psychometric properties in Chinese patients with BC. ${ }^{51}$

\section{DATA COLLECTION, MANAGEMENT AND ANALYSIS \\ Data collection}

The participants' baseline information will be collected via a self-designed baseline data assessment form and extracted from the participants' medical records at baseline (T1) before randomisation. All the feasibility outcomes will be recorded throughout the study process and computed immediately after the completion of the intervention (T2). However, adverse events of the SA interventions will be assessed and managed in a timely 
manner throughout the intervention period. All the clinical outcomes will be assessed at two time points: baseline (T1) before randomisation and immediately after the completion of the intervention (T2). Paper questionnaires will be used to collect all the clinical outcomes via face-to-face or telephone survey by the research assistant. As all the questionnaires will be self-administrated, the participants will be encouraged to complete the questionnaires by themselves. Assistance will only be provided when necessary, for example, providing a neutral interpretation if the participants feel confused about terms or items in the questionnaires, and providing assistance to participants with vision problems through reading the items aloud word by word.

\section{Data management}

All hard copies of this study will be kept in a locked cabinet in the Southwest Medical University Hospital (study site investigator's office), strictly following the university's regulations and policies. All electronic data will be stored in a secure research hard drive with password-protected access systems. On the completion of the study, a copy of the electronic files will be provided to the principal investigator via a zipped folder that can only be accessed by the principal investigator and the study site investigator via passwords. All the datasets will be double-checked against the paper recordings of raw data to ensure that the data coding is correct. Data cleaning will be carried out to maintain the validity of the statistical analysis, including checking for incorrectly entered data, missing data, and outliers.

\section{Confidentiality}

Information collected from the participants will be used for research purposes only. To ensure that the research team will manage the data in a confidential and anonymous way, every document collected from the participants will be recorded using an identification code rather than using the participants' name or other clear identifiers.

\section{Statistical methods}

Descriptive statistics will be used to present the feasibility outcomes (eg, eligibility rate, recruitment rate and retention rate). The results of the feasibility outcomes will be used to determine whether the main study can proceed or not. ${ }^{52}$ If all the criteria are achieved, the main study will proceed without modifications; if most or some criteria are achieved, the main study will proceed with modifications; and if only a few or no criteria are achieved, the main study will not proceed.$^{52}$ The $\chi^{2}$ test or Fisher's exact test will be utilised to identify the between-group differences for categorical variables. A one-way (or Welch) analysis of variance (ANOVA) or Kruskal-Wallis test will be used to for continuous variables. The clinical outcomes will be analysed based on the principle of intention-to-treat analysis, and the missing data will be handled via the approach of last observation carried forward. The symptom cluster will be measured using the approach of transferring the total score of each single symptom scale (MFI, PSQI and HADS-D) into a $0-10$ Numerical Rating Scale $(0=$ 'no', $10=$ 'as bad as it could be'). Then, symptom severity at the cluster level will be evaluated by calculating the average scores of the three symptoms on 0-10 Numerical Rating Scales. ${ }^{2753}$ The effects of SA on the symptom cluster and each single symptom (fatigue, sleep disturbance, depression and QoL) will be analysed using either parametric analysis or non-parametric analysis based on the distribution of the data and the homogeneity of variance. If all these assumptions are not violated, a one-way ANOVA will be conducted; if the homogeneity assumption is violated, the Welch ANOVA will be used. Otherwise, the Kruskal-Wallis test will be utilised. For comparisons with statistically significant differences via ANOVA, a post-hoc comparison will be conducted using Tukey's test (homogeneity assumption achieved) or the Games-Howell test (homogeneity assumption violated) to examine the between-group differences. To control the confounding effects of potential factors on the FSD symptom cluster, the among-group effects of SA on fatigue, sleep disturbance, depression and QoL will be additionally analysed using the generalised estimating equation model. Effect size and statistical power for the between-group comparisons of SA on fatigue, sleep disturbance, depression and QoL will be computed using Cohen's $d$, which will be used to estimate the sample size in a future large-scale RCT. Thematic analysis will be used to analyse the qualitative data, and relevant results will be used to further support and supplement the quantitative findings.

\section{DISCUSSION}

The FSD symptom cluster, a common and long-term distressing problem in BC survivors, can considerably impact patients' function status and QoL throughout the cancer trajectory. ${ }^{55}$ Current research evidence has been limited to recommending promising pharmaceutical agents to manage the FSD symptom cluster. Non-pharmacological interventions have gradually drawn clinicians' and cancer survivors' attention as a combination treatment approach to alleviate cancer-related symptoms. SA is a promising non-pharmacological approach that has been frequently utilised in research and practice and that is easily accepted by patients. Existing research has supported the promising role of acupoint stimulation for cancer symptom management. However, most studies have focused on individual symptoms only, ${ }^{212} 2324$ not the entire symptom cluster. The proposed study will follow the MRC Framework to examine an evidence-based SA protocol for the self-management of the FSD symptom cluster in BC survivors by comprehensively examining its clinical feasibility and effectiveness, all of which can inform clinical decision making and healthcare policy-making regarding the utilisation of SA in practice and contribute to the evidence base for self-managing cancer symptoms in the long run.

The MRC Framework-based study design will ensure that the development of the SA intervention protocol 
will be evidence based and research informed, and the acceptability and feasibility of the SA intervention as well as the research methodological procedures will be fully determined prior to a phase III definitive RCT. The development of the SA intervention protocol, including the selection of acupoints formula, modality, intensity, technique and dosage (frequency, sessions and total duration), will be rigorously developed based on widely recognised theories, best available research evidence identified from well-conducted systematic reviews and practice standards/ handbooks and the consensus of an expert panel. Using such an evidence-based and research-informed method to develop the SA intervention protocol will be the key strength of the proposed study. The design of the sham SA comparison will be another strength of the study, which will enable the measurement of potential placebo effects by comparing the true SA intervention with the sham SA intervention. The size of the placebo effects of the sham SA intervention will also be identified through a further comparison between the sham SA group and the usual care group.

This study has some limitations and challenges. Although the participants will perform SA in a separate space (at their own home), it will still be challenging to maintain a successful blind design among the participants due to the visible nature of the intervention. To further minimise the risk of breaking the blind design, potential participants with any previous acupoint stimulation treatment experience during the past 6 months will be excluded. As a self-administered intervention, how to maintain consistency in practising SA among the participants and improve the participants' compliance with regular SA will be a challenge in this study. Before the intervention, standardised training on the SA intervention protocol will be provided to the participants in the true and sham SA groups by the same research assistant, and the participants' skills will be evaluated via return demonstration after the training. Regular telephone follow-ups by the research assistant and daily log recording by the study participants will be used to enhance the participants' adherence to the intervention.

This study protocol reported the overall design and methodological procedures of the phase II RCT, which aims to evaluate the feasibility and potential effects of an evidencebased SA intervention for the management of the FSD symptom cluster in BC survivors. The findings from this study will inform a well-established SA intervention that can be further utilised in a phase III multisite RCT to confirm the definitive effects of SA on the FSD symptom cluster and QoL in BC survivors.

\section{Author affiliations}

${ }^{1}$ College of Nursing and Midwifery, Charles Darwin University, Brisbane, Queesland, Australia

${ }^{2}$ College of Nursing and Midwifery, Charles Darwin University, Darwin, Northern Territory, Australia

${ }^{3}$ Department of Nursing, Affiliated Hospital of Southwest Medical University, Luzhou, Sichuan, China

${ }^{4}$ Cancer and Palliative Outcomes Centre, Queensland University of Technology, Brisbane, Queensland, Australia

${ }^{5}$ Thornlands General Practice, Brisbane, Queensland, Australia
${ }^{6}$ School of Nursing, The Hong Kong Polytechnic University, Kowloon, Hong Kong SAR

Contributors TW: study design and manuscript drafting and revision; J-YBT: study conception and design, and manuscript revision; X-LL, IZ, SE, MJP and HC: study design and manuscript revision; S-LZ and $\mathrm{H}-\mathrm{QH}$ : study coordination and manuscript revision.

Funding This study is supported by the IAS Rainmaker Readiness Grant at Charles Darwin University, Australia.

Disclaimer The funder will not be involved in the study design, data collection, management, analysis and interpretation, report writing, and the decision to submit the report for publication.

Competing interests None declared.

Patient consent for publication Consent obtained directly from patient(s) Provenance and peer review Not commissioned; externally peer reviewed.

Supplemental material This content has been supplied by the author(s). It has not been vetted by BMJ Publishing Group Limited (BMJ) and may not have been peer-reviewed. Any opinions or recommendations discussed are solely those of the author(s) and are not endorsed by BMJ. BMJ disclaims all liability and responsibility arising from any reliance placed on the content. Where the content includes any translated material, BMJ does not warrant the accuracy and reliability of the translations (including but not limited to local regulations, clinical guidelines, terminology, drug names and drug dosages), and is not responsible for any error and/or omissions arising from translation and adaptation or otherwise.

Open access This is an open access article distributed in accordance with the Creative Commons Attribution Non Commercial (CC BY-NC 4.0) license, which permits others to distribute, remix, adapt, build upon this work non-commercially, and license their derivative works on different terms, provided the original work is properly cited, appropriate credit is given, any changes made indicated, and the use is non-commercial. See: http://creativecommons.org/licenses/by-nc/4.0/.

\section{ORCID iDs}

Tao Wang http://orcid.org/0000-0001-9845-3988

Jing-Yu (Benjamin) Tan http://orcid.org/0000-0002-1609-6890

Hui-Lin Cheng http://orcid.org/0000-0002-6117-3832

\section{REFERENCES}

1 Harbeck N, Penault-Llorca F, Cortes J, et al. Breast cancer. Nat Rev Dis Primers 2019;5:66.

2 Leong E, Madli F, Ong SK. Five-year survival rate of breast cancer patients in Brunei Darussalam. Brunei International Medical Journal 2019;15:73-81.

3 McFarland DC, Shaffer KM, Tiersten A, et al. Physical symptom burden and its association with distress, anxiety, and depression in breast cancer. Psychosomatics 2018;59:464-71.

4 Peoples AR, Roscoe JA, Block RC, et al. Nausea and disturbed sleep as predictors of cancer-related fatigue in breast cancer patients: a multicenter NCORP study. Supportive Care in Cancer 2017;25:1271-8.

5 Berger AM, Mooney K, Alvarez-Perez A, et al. Cancer-related fatigue, version 2.2015. J Nat/ Compr Canc Netw 2015;13:1012-39.

6 Bower JE. Cancer-related fatigue-mechanisms, risk factors, and treatments. Nat Rev Clin Oncol 2014;11:597-609.

7 Accortt EE, Bower JE, Stanton AL, et al. Depression and vasomotor symptoms in young breast cancer survivors: the mediating role of sleep disturbance. Arch Womens Ment Health 2015;18:565-8.

8 Bower JE, Ganz PA, Irwin MR, et al. Inflammation and behavioral symptoms after breast cancer treatment: do fatigue, depression, and sleep disturbance share a common underlying mechanism? Journal of Clinical Oncology 2011;29:3517-22.

9 Colagiuri B, Christensen S, Jensen AB, et al. Prevalence and predictors of sleep difficulty in a national cohort of women with primary breast cancer three to four months postsurgery. J Pain Symptom Manage 2011;42:710-20.

10 Reinertsen KV, Engebraaten O, Loge $\mathrm{JH}$, et al. Fatigue during and after breast cancer therapy - a prospective study. J Pain Symptom Manage 2017;53:551-60.

11 Ho S-H, Rohan KJ, Parent J. A longitudinal study of depression, fatigue, and sleep disturbances as a symptom cluster in women with breast cancer. J Pain Symptom Manage 2015;49:707-15.

12 Trudel-Fitzgerald C, Savard J, Ivers H. Which symptoms come first? Exploration of temporal relationships between cancer- 
related symptoms over an 18-month period. Ann Behav Med 2013;45:329-37.

13 Irwin MR. Depression and insomnia in cancer: prevalence, risk factors, and effects on cancer outcomes. Curr Psychiatry Rep 2013;15:404

14 Dodd MJ, Cho MH, Cooper BA, et al. The effect of symptom clusters on functional status and quality of life in women with breast cancer. Eur J Oncol Nurs 2010;14:101-10.

15 Fiorentino L, Rissling M, Liu L, et al. The symptom cluster of sleep, fatigue and depressive symptoms in breast cancer patients: severity of the problem and treatment options. Drug Discov Today Dis Models 2011;8:167-73.

16 Induru RR, Walsh D. Cancer-related insomnia. Am J Hosp Palliat Care 2014;31:777-85.

17 Sanford SD, Beaumont JL, Butt Z, et al. Prospective longitudinal evaluation of a symptom cluster in breast cancer. J Pain Symptom Manage 2014;47:721-30.

18 Innominato PF, Lim AS, Palesh O, et al. The effect of melatonin on sleep and quality of life in patients with advanced breast cancer. Supportive Care in Cancer 2016;24:1097-105.

19 Madsen MT, Hansen MV, Andersen LT, et al. Effect of melatonin on sleep in the perioperative period after breast cancer surgery: a randomized, double-blind, placebo-controlled trial. Journal of Clinical Sleep Medicine 2016;12:225-33.

20 Choi T-Y, Kim Jl, Lim H-J, et al. Acupuncture for managing cancerrelated insomnia: a systematic review of randomized clinical trials. Integr Cancer Ther 2017;16:135-46.

21 Finnegan-John J, Molassiotis A, Richardson A, et al. A systematic review of complementary and alternative medicine interventions for the management of cancer-related fatigue. Integr Cancer Ther 2013;12:276-90.

22 Rieger KL, Lobchuk MM, Duff MA, et al. Mindfulness-based arts interventions for cancer care: a systematic review of the effects on wellbeing and fatigue. Psychooncology 2021;30:240-51.

23 Kumar JVR, Uma M. Effectiveness of acupressure on improving the quality of sleep among cancer patients. Asian Journal of Nursing Education and Research 2015;5:513-7.

24 Wang T, Deng R, Tan J-Y, et al. Acupoints stimulation for anxiety and depression in cancer patients: a quantitative synthesis of randomized controlled trials. Evid Based Complement Alternat Med 2016;2016:1-15.

25 So WK, Law BM, Chan DN. The effect of nonpharmacological interventions on managing symptom clusters among cancer patients: a systematic review. Cancer Nurs 2020;43:E304-27.

26 Cheung DS, Yeung WF, Chau PH. Patient-centred, self-administered acupressure for Chinese advanced cancer patients experiencing fatigue and co-occurring symptoms: a pilot randomised controlled trial. Eur J Cancer Care 2020;7:e13314.

27 Hoang HT, Molassiotis A, Chan CW. Pilot randomized shamcontrolled trial of self-acupressure to manage the symptom cluster of insomnia, depression, and anxiety in cancer patients undergoing chemotherapy. Sleep Breathing 2021;14:1-2

28 Craig P, Dieppe P, Macintyre S, et al. Developing and evaluating complex interventions: the new medical Research Council guidance. Int J Nurs Stud 2013;50:587-92.

29 Chan A-W, Tetzlaff JM, Altman DG, et al. Spirit 2013 statement: defining standard protocol items for clinical trials. Ann Intern Med 2013;158:200-7.

30 Julious SA. Sample size of 12 per group rule of thumb for a pilot study. Pharm Stat 2005;4:287-91.

31 Zick SM, Sen A, Wyatt GK, et al. Investigation of 2 types of selfadministered acupressure for persistent cancer-related fatigue in breast cancer survivors. JAMA Oncol 2016;2:1470-6.

32 Vickers AJ, Cronin AM, Maschino AC. Acupuncture for chronic pain: individual patient data meta-analysis. Arch Intern Med 2012;172:1444-53.

33 Liu X-L, Cheng HL, Moss S, et al. Somatic acupoint stimulation for cancer-related sleep disturbance: a systematic review of randomized controlled trials. Evid Based Complement Alternat Med 2020;2020:1-12.

34 Tan J-YB, Wang T, Kirshbaum MN, et al. Acupoint stimulation for cancer-related fatigue: a quantitative synthesis of randomised controlled trials. Complement Ther Clin Pract 2021;45:101490.

35 Liang FR, Wang H. Acupuncture and moxibustion. 4th ed. Beijing: China Press of Traditional Chinese Medicine Co. Ltd, 2016.

36 Shen XY. Meridians and acupoints. 4th ed. Beijing: China Press of Traditional Chinese Medicine Co. Ltd, 2008.

37 Zick SM, Alrawi S, Merel G, et al. Relaxation acupressure reduces persistent cancer-related fatigue. Evid Based Complement Alternat Med 2011;2011:1-10.

38 Tan J-Y, Suen LKP, Wang T, et al. Sham acupressure controls used in randomized controlled trials: a systematic review and critique. PLoS One 2015;10:e0132989.

39 Dincer F, Linde K. Sham interventions in randomized clinical trials of acupuncture-a review. Complement Ther Med 2003;11:235-42.

40 National Comprehensive Cancer Network Cancer-Related Fatigue Panel. National comprehensive cancer network clinical practice guidelines in oncology. cancer-related fatigue, version 2, 2018.

41 Department of health at the government of Western Australia. Available: https://www.health.gov.au/resources

42 Berger AM, Mitchell SA, Jacobsen PB, et al. Screening, evaluation, and management of cancer-related fatigue: ready for implementation to practice? CA Cancer J Clin 2015;65:190-211.

43 Li M, Fitzgerald P, Rodin G. Evidence-based treatment of depression in patients with cancer. J Clin Oncol 2012;30:1187-96.

44 Bellg AJ, Borrelli B, Resnick B, et al. Enhancing treatment fidelity in health behavior change studies: best practices and recommendations from the NIH behavior change Consortium. Health Psychol 2004;23:443-51.

45 Smith SW, Daunic AP, Taylor GG. Treatment fidelity in applied educational research: expanding the adoption and application of measures to ensure evidence-based practice. Education and Treatment of Children 2007;30:121-34.

46 Tan J. Effects of auricular acupressure on chemotherapy-induced nausea and vomiting in breast cancer patients: a preliminary randomized controlled trial [PhD thesis]. The Hong Kong Polytechnic University, 2017

47 Tian J, Hong J-S. Validation of the Chinese version of multidimensional fatigue Inventory-20 in Chinese patients with cancer. Supportive Care Cancer 2012;20:2379-83.

48 Buysse DJ, Reynolds CF, Monk TH, et al. The Pittsburgh sleep quality index: a new instrument for psychiatric practice and research. Psychiatry Res 1989;28:193-213.

49 Ho RT, Fong TC. Factor structure of the Chinese version of the Pittsburgh sleep quality index in breast cancer patients. Sleep Med 2014;15:565-9.

50 Li Q, Lin Y, Hu C, et al. The Chinese version of hospital anxiety and depression scale: psychometric properties in Chinese cancer patients and their family caregivers. Eur $\mathrm{J}$ Oncol Nurs 2016;25:16-23

51 Wan C, Zhang D, Yang Z, et al. Validation of the simplified Chinese version of the FACT-B for measuring quality of life for patients with breast cancer. Breast Cancer Res Treat 2007;106:413-8.

52 Thabane L, Ma J, Chu R, et al. A tutorial on pilot studies: the what, why and how. BMC Med Res Methodol 2010;10.

53 Nguyen LT, Alexander K, Yates P. Psychoeducational intervention for symptom management of fatigue, pain, and sleep disturbance cluster among cancer patients: a pilot quasi-experimental study. $J$ Pain Symptom Manage 2018;55:1459-72.

54 Fitzgerald P, Lo C, Li M, et al. The relationship between depression and physical symptom burden in advanced cancer. BMJ Support Palliat Care 2015;5:381-8.

$55 \mathrm{Oh} \mathrm{H}$, Seo $\mathrm{Y}$, Jeong $\mathrm{H}$, et al. The identification of multiple symptom clusters and their effects on functional performance in cancer patients. J Clin Nurs 2012;21:2832-42. 